\title{
Gender-based analysis using existing public health datasets
}

\section{L'analyse du genre à l'aide d'ensembles de données de santé publique existants}

\author{
Cara Tannenbaum ${ }^{1}$ \\ Published online: 12 March 2020 \\ (C) The Canadian Public Health Association 2020
}

On December 13, 2019, the Prime Minister of Canada released new Ministerial Mandate letters, setting a high bar for inclusive and accountable public health evidence-based decision-making (Trudeau 2019). The letters assert that genderbased analyses must be applied to all federal programs and policies. Biological sex-as well as the more socially driven concept of gender-are to be cemented as cornerstones of achieving equitable outcomes in this country. Our research and analyses should be poised to deliver on this promise.

To some public health researchers and practitioners, the directive seems redundant. After all, the Government of Canada released its sex and gender-based analysis policy 10 years earlier in 2009 to ensure that programs and services address the different needs of diverse groups of women and men (Government of Canada 2009). Most public health analyses already disaggregate data by sex, age, and race. For other public health researchers, however, the call to action was long overdue. The lack of implementation of the Canadian sex and gender-based analysis policy in the spirit it was originally intended contributes daily to the persistence of health disparities. The policy has remained in effect for over a decade, but it is far from being alive and well.

Thriving applications of sex and gender-based analysis envision every analyst disaggregating their data by sex, and also being able to define the role gender plays in explaining subgroup disparities. Researchers would consistently and competently distinguish biological sex from various components of gender-gender roles, gender identities, and gender relations - in their analyses, reports, and discussions, and

Cara Tannenbaum

cara.tannenbaum@umontreal.ca

1 CIHR Institute of Gender and Health, Montreal, QC, Canada know which component(s) of gender is/are most relevant to the program or policy under consideration. The design of community-based programs and services would be genderresponsive, based on consideration of inequities and a view towards redressing the systemic underlying causes (Tannenbaum et al. 2016). More importantly, evaluative frameworks would be in place to regularly monitor and track whether the outcomes of gender-responsive programs are successful in reducing risk factors or narrowing outcome gaps for specific populations over time.

In an era of real-world evidence and continuous learning systems, one could reflect on why we failed to do things right back in 2009 and how to guarantee we do the right things going forward. Increased knowledge and capacity for conducting sex and gender-based analyses within the public health sector is an obvious first step. Public health researchers and practitioners require a better understanding of gender in all its multifaceted complexity-as distinct from biological sex. Also on the roster is the need for a plan to routinely collect data on gender roles, gender identities, and gender relations in future quantitative and qualitative studies. All analysts should be competent in analyzing gender even when existing datasets omit primary measures of gender. Finally, capacity must be built for interpreting gender-based analyses in a way that thoughtfully and concretely informs policies and programs tailored to gender.

Lacasse et al. (2020) tackle the challenge of developing methods for analyzing gender in datasets that a priori do not collect data on gender. The authors scoured three iterations of the Canadian Community Health Survey (CCHS) for variables that might serve as proxy representations of gender and be included in a gender index. Lacasse et al. identified 50 candidate variables for deriving the gender index. Many of these variables captured aspects of societally determined gender roles and behaviours, such as being the person the most 
knowledgeable about the household situation, caring for children as the most important thing contributing to feelings of stress, or the probability of being the driver of, or passenger in, a snowmobile, motor boat, seadoo, or all-terrain vehicle in the past 12 months. Regrettably, only 19 of the 50 variables were measured in all three iterations of the CCHS, had less than $15 \%$ missing values, and did not experience collinearity issues, leaving data from 29,470 participants aged $18-50$ years eligible for inclusion in the analysis.

The remaining 19 variables underwent testing for the gender index through multivariable regression against sex and using propensity score analysis. The six variables with the highest regression coefficients were (1) having an occupation in the field of trades, transport, and equipment operators; (2) receiving child support as the main source of household income; (3) working in an organization of the healthcare or social assistance sector; (4) having an occupation in the field of health, social science, education, government service, or religion; (5) working in an organization of the construction or manufacturing sector; and (6) number of working hours per week.

The investigators should be applauded for the rigour they applied to creating the gender index. Gender experts were involved in selecting gender-related candidate variables, the index underwent appropriate statistical modeling, and psychometric testing was conducted to establish construct validity. Future studies will determine whether the gender index has predictive power and whether it is generalizable to public health datasets other than those derived from the CCHS. The good news is that important precedents have been set by other research groups using similar methods to create gender scores. Smith and Koehoorn (2016) worked with data from the Canadian Labour Force survey to derive a 4-item gender index with parallel variables: responsibility for caring for children; occupation segregation; hours of work; and level of education. Pelletier et al. (2016) created a more complex 7-item gender score from the GENESIS-PRAXY cardiovascular dataset and discovered that gender, independent of sex, predicted poor outcomes after early coronary syndrome, opening up novel possibilities for future interventions. The Canadian Institutes of Health Research offers an online learning module for data analysts who are keen to hone their sex and gender skills using large administrative datasets (available at https://www.cihr-irsc-igh-isfh.ca/?lang=en).

Lest the effort appear too daunting to conduct a proper gender-based analysis using existing datasets, consider the success story of Sweden's gender-balanced snow-clearing policy (Swedish Association of Local Authorities and Regions 2019). Analysis of health claim datasets on fallrelated injuries during the winter season revealed that women were more likely to slip on the ice while traversing city routes essential for gendered occupational activities such as caring for children or accessing hospitals. These city routes involved sidewalks and small arteries that had delayed snow-clearing compared to the larger, more traffic-heavy car-driving routes that delivered workers to downtown business areas. In the spirit of gender-balanced safe cities and environments, a new snow-clearing policy was implemented in Sweden that temporally prioritized snow-clearing for routes more frequently used by women in the course of their gender-related activities. The outcome was a dramatic decline in falls and injuries during the next snow-clearing season.

As we break new ground in applying gender-based analyses to public health research, programs, and policies, some cautionary advice is warranted. Keep in mind the goal of discovering how gender contributes to health disparities and what we can do to avoid the unintended consequences of policies and programs that neglect gender. Do not be distracted by the need to create different gender indices for every dataset. Instead look for common variables and concepts that can be generalized across multiple data sources and populations for comparative purposes. Remember that accounting for intersectionality means seeking out the voices of individuals and communities who experience simultaneous layers of power imbalance due to gender, education, geography, socioeconomic status, race, and religion. Objective data on gender will always be required for evidence-based decision-making, but no amount of quantitative manipulation will ever surpass the richness of conversation and storytelling for probing potential public health solutions. Gender-based analysis using existing public health datasets is indeed emerging as a necessary tool for informing better programs and policies, but to reap its benefits, appropriate application and evaluation is required to best serve the health of those populations who need it most.

\section{Cara Tannenbaum, Scientific Director}

CIHR Institute of Gender and Health

\section{Éditorial}

Le 13 décembre 2019, le premier ministre du Canada a remis à ses ministres de nouvelles lettres de mandat qui prévoient des processus décisionnels de santé publique fondés sur les résultats nettement plus inclusifs et responsables (Trudeau 2019). Ces lettres affirment que des analyses du genre doivent être appliquées à l'ensemble des politiques et des programmes fédéraux. Le sexe biologique et la notion de genre - à caractère plus social - doivent devenir les pierres angulaires de l'atteinte de résultats équitable au pays. Nos études et nos analyses doivent être en mesure de tenir cette promesse.

Pour certains chercheurs et praticiens en santé publique, c'est une directive qui peut sembler redondante. Après tout, le gouvernement du Canada a publié sa politique sur l'analyse des différences de genre et des différences de sexe il y a déjà 
10 ans, en 2009, pour que les programmes et les services tiennent compte de divers groupes de femmes et d'hommes et de leurs besoins (Gouvernement du Canada 2009). La plupart des analyses de santé publique décomposent déjà les données selon le sexe, l'âge et la race. Pour d'autres chercheurs en santé publique toutefois, cet appel à l'action était attendu depuis longtemps. La non-application de la politique canadienne sur l'analyse des différences de genre et des différences de sexe dans l'esprit qui devait être la sienne contribue tous les jours à la persistance des disparités d'état de santé. La politique a beau être en vigueur depuis plus de 10 ans, elle est loin d'être florissante.

Dans les applications fécondes de l'analyse des différences de sexe et des différences de genre, chaque analyste décomposerait ses données selon le sexe et serait capable de définir le rôle du genre pour expliquer les disparités entre les sous-groupes. Les chercheurs distingueraient systématiquement et avec compétence le sexe biologique des diverses composantes du genre - les rôles de genre, les identités de genre et les relations entre les genres - dans leurs analyses, leurs rapports et leurs discussions et sauraient laquelle ou lesquelles de ces composantes conviennent le mieux au programme ou à la politique à l'étude. Dans leur conception, les programmes et les services de proximité seraient différenciés selon le genre et tiendraient compte des inégalités en vue d'en corriger les causes systémiques sous-jacentes (C. Tannenbaum, L. Greaves et I.D. Graham 2016). Mais surtout, il y aurait des cadres d'évaluation pour suivre régulièrement les résultats des programmes différenciés selon le genre et voir s'ils réussissent à réduire les facteurs de risque ou les écarts dans les résultats de certaines populations au fil du temps.

À l'ère des preuves concrètes et des systèmes d'apprentissage continu, on peut se demander pourquoi nous n'avons pas fait correctement les choses en 2009 et comment garantir que nous ferons mieux à partir de maintenant. L'accroissement des connaissances et des capacités nécessaires pour mener des analyses des différences de sexe et des différences de genre dans le secteur de la santé publique est un premier pas logique. Les chercheurs et les praticiens en santé publique ont besoin de mieux comprendre le genre sous toutes ses facettes, par opposition au sexe biologique. Ajoutons à la liste un plan de collecte systématique de données sur les rôles de genre, les identités de genre et les relations entre les genres dans les futures études quantitatives et qualitatives. Tous les analystes devraient être compétents en matière d'analyse du genre, même quand les ensembles de données existants omettent les indicateurs primaires du genre. Enfin, il faut nous doter d'une capacité d'interpréter de façon concrète et réfléchie les analyses du genre pour éclairer les politiques et les programmes adaptés au genre.

Lacasse et collègues (2020) s'attaquent au défi d'élaborer des méthodes d'analyse du genre dans des ensembles de données qui a priori ne collectent pas d'informations sur le genre. Les auteurs ont écumé trois itérations de l'Enquête sur la santé dans les collectivités canadiennes (ESCC) pour trouver des variables pouvant servir de représentations approximatives du genre afin de les inclure dans un « indice de genre ». Ils ont répertorié 50 variables candidates pour cet indice de genre. Beaucoup de ces variables saisissent des aspects des rôles et des comportements de genre déterminés par la société, comme le fait d’être la personne qui connaît le mieux la situation du ménage, le soin des enfants comme principal facteur contribuant aux sentiments de stress, ou la probabilité d'avoir été conducteur ou passager d'une motoneige, d'un bateau à moteur, d'une motomarine ou d'un véhicule tout-terrain au cours des 12 mois antérieurs. Malheureusement, seulement 19 des 50 variables ont été mesurées dans les trois itérations de l'ESCC, comportaient moins de $15 \%$ de valeurs manquantes et n'avaient pas de problèmes de colinéarité, ne laissant que les données de 29470 participants de 18 à 50 ans à inclure dans l'analyse.

Les 19 variables restantes ont été testées pour l'indice de genre au moyen d'une régression multivariée par rapport au sexe et d'une analyse du score de propension. Les six variables dont les coefficients de régression étaient les plus élevés sont : 1) le fait d'avoir une profession dans le domaine des métiers, des transports ou de la conduite de machinerie, 2) le fait qu'une pension alimentaire pour enfants soit la principale source de revenu du ménage, 3) l'emploi dans un organisme du secteur des soins de santé ou de l'aide sociale, 4) le fait d'avoir une profession dans le domaine de la santé, des sciences sociales, de l'éducation, des services gouvernementaux ou de la religion, 5) l'emploi dans un organisme du secteur du bâtiment ou de la fabrication et 6) le nombre d'heures de travail par semaine.

Les chercheurs devraient être applaudis pour la rigueur avec laquelle ils ont créé leur indice de genre. Des spécialistes du genre ont participé à la sélection des variables candidates liées au genre, l'indice a fait l'objet d'une modélisation statistique appropriée, et des tests psychométriques ont été menés pour établir la validité conceptuelle. De futures études détermineront si l'indice de genre détient un pouvoir prédictif et s'il peut être généralisé aux ensembles de données de santé publique autres que ceux dérivés de l'ESCC. La bonne nouvelle est que d'importants précédents ont été établis par d'autres groupes de recherche utilisant des méthodes semblables pour créer des indices de genre. Smith et Koehoorn (2016) ont travaillé avec les données de l'Enquête canadienne sur la population active pour dériver un indice de genre à 4 éléments avec des variables parallèles : la responsabilité du soin des enfants, la ségrégation professionnelle, les heures de travail et le niveau d'instruction. Pelletier et collègues (2016) ont créé un indice de genre plus complexe, à 7 éléments, à partir de l'ensemble de données cardiovasculaires de l'étude GENESIS-PRAXY, et ont découvert que le genre, indépendamment du sexe, permet de 
prédire des résultats défavorables après un syndrome coronarien précoce, ce qui ouvre de nouvelles possibilités à des interventions futures. Les Instituts de recherche en santé du Canada offrent un module d'apprentissage en ligne aux analystes de données qui cherchent à parfaire leurs compétences en matière de sexe et de genre à l'aide de grands ensembles de données administratives (ce module est accessible sur https://www.cihr-irsc-igh-isfh.ca/?lang=fr).

Si l'effort nécessaire pour mener de bonnes analyses du genre à l'aide des ensembles de données existants vous paraît insurmontable, pensez à la Suède, dont la politique de déneigement respecte l'équilibre entre les sexes (Association suédoise des collectivités locales et régionales 2019). Une analyse des ensembles de données de réclamations d'assurance-santé pour blessures causées par des chutes durant la saison hivernale avait montré que les femmes étaient plus susceptibles de glisser sur la glace lorsqu'elles empruntaient les voies urbaines essentielles aux activités professionnelles genrées, comme l'accès aux garderies et aux hôpitaux. Ces voies urbaines comportent des trottoirs et de petites artères dont le déneigement se faisait après celui des voies de circulation automobile, plus larges et à haute densité de trafic, qui transportent les travailleurs vers les quartiers des affaires des centres-villes. Pour avoir des villes et des environnements sûrs qui respectent l'équilibre entre les sexes, la Suède a donc appliqué une nouvelle politique de déneigement qui accorde la priorité temporelle au déneigement des voies plus souvent empruntées par les femmes au cours de leurs activités genrées. Il en est résulté une baisse spectaculaire des chutes et des blessures durant la saison de déneigement suivante.

Avant d'innover en appliquant des analyses du genre à la recherche, aux programmes et aux politiques en santé publique, la prudence est de mise. Gardons en tête que l'objectif est de découvrir comment le genre contribue aux disparités d'état de santé et ce que nous pouvons faire pour éviter les effets pervers des politiques et des programmes qui négligent l'aspect du genre. Ne nous laissons pas distraire par le besoin de créer un nouvel indice de genre pour chaque ensemble de données. Attardons-nous plutôt aux variables et aux concepts courants qui peuvent être étendus à plusieurs sources de données et à plusieurs populations à des fins de comparaison. N'oublions pas que tenir compte de l'intersectionnalité signifie chercher à entendre les personnes et les populations aux prises avec des niveaux simultanés de déséquilibre de pouvoir dus au genre, à l'instruction, à la géographie, au statut socioéconomique, à la race et à la religion. Des données objectives sur le genre seront toujours nécessaires à la prise de décisions fondée sur les résultats, mais aucune manipulation des chiffres ne surpassera jamais la richesse de la conversation et de la narration pour explorer d'éventuelles solutions de santé publique. Il ne fait aucun doute que les analyses du genre qui font appel aux ensembles de données de santé publique existants sont en train de devenir des outils nécessaires pour éclairer et améliorer les programmes et les politiques, mais pour en récolter les bénéfices, il faut les appliquer et les évaluer convenablement, pour le bien de la santé des populations qui en ont le plus besoin.

Cara Tannenbaum, Directrice scientifique

IRSC Institut de la santé des femmes et des hommes

\section{References/Références bibliographiques}

Government of Canada. (2009). Sex and gender-based analysis policy. Available at https://www.canada.ca/en/health-canada/corporate/ transparency/corporate-management-reporting/heath-portfolio-sexgender-based-analysis-policy.html (Accessed December 22, 2019).

Lacasse, A., Pagé, M. G., Choinière, M., \& on behalf of the TORSADE Cohort Working Group. (2020). Conducting gender-based analysis of existing databases when self-reported gender data are unavailable: the GENDER Index in a working population. Canadian Journal of Public Health, 111(2). https://doi.org/10.17269/s41997019-00277-2.

Pelletier, R., Khan, N. A., Cox, J., Daskalopoulou, S. S., Eisenberg, M. J., Bacon, S. L., et al. (2016). Sex versus gender-related characteristics: which predicts outcome after acute coronary syndrome in the young? Journal of the American College of Cardiology, 67(2), 127-135.

Smith, P. M., \& Koehoorn, M. (2016). Measuring gender when you do not have a gender measure: constructing a gender index using survey data. International Journal for Equity in Health, 15, 82.

Swedish Association of Local Authorities and Regions. Sustainable gender equality - a film about gender mainstreaming in practice. Available at https://www.youtube.com/watch?v=udSjBbGwJEg (Accessed December 23, 2019).

Tannenbaum, C., Greaves, L., \& Graham, I. D. (2016). Why sex and gender matter in implementation research. BMC Medical Research Methodology, 16(1), 145.

Trudeau J, Prime Minister of Canada. (2019). Mandate letters. Available at https://pm.gc.ca/en/mandate-letters (Accessed December 22, 2019).

Publisher's note Springer Nature remains neutral with regard to jurisdictional claims in published maps and institutional affiliations. 